\title{
Treatment of impacted lower left second molar and over erupted upper second molar
}

\begin{abstract}
This article describes a case report of impacted lower left second molar. A 22 year old female patient was found with over erupted upper second right molar, reaching the edge of the lower alveolar ridge. The reason for this was an impacted lower left second molar. Our treatment went through the following stages: first with the intrusion of the upper left second molar, which made a space in vertical direction for the guided traction of the lower left second molar; then we uprighted and positioned the lower second molar in the dental arch and normalized occlusal ratios. The patient was pleased with the achieved result. We restored aesthetics in the frontal segment as well as the function in the distal segment. It is crucial to make an early diagnosis, to choose optimal treatment time and reduction of complications. The mesial tipping of the impaction and the depth of impaction has more important roles than patient's age and the difficulty of treatment.
\end{abstract}

Keywords: Lower second molar, impacted teeth, uprighting molar, unerupted tooth, orthodontic treatment
Volume 9 Issue 2 - 2018

\author{
Greta Yordanova \\ Department of Orthodontic, Medical University, Bulgaria
}

Correspondence: Greta Yordanova, DDS, PhD Department of Orthodontic, Faculty of Dental Medicine, Medical University, I, St.Georgi Sofiiski, I 608 Sofia, Bulgaria, Tel 00359877922665 , Email gretayordanova@gmail.com

Received: February 12, 2018 | Published: March 05, 2018

\section{Introduction}

Tooth impaction is defined as any tooth that fails to erupt into a normal functional position and remains unerupted in the jaw beyond the time when it should normally erupt. ${ }^{1}$ Impaction of a permanent tooth is a relatively common clinical occurrence that may involve any tooth in the human dentition. Primary retention of molars occurs before the eruption and the cause could be either space problems or failure in the dental follicle's ability to initiate resorption of the overlying bone. ${ }^{2-4}$ Impaction of the mandibular permanent second molars are not very common and their prevalence studied either clinically or by inspection of the panoramic radiographs has been reported to be $0.03 \%$ of the general population and $2-3 \%$ of the orthodontic patients. ${ }^{5,6}$ Bondemark and Fu reported that the impaction of a second permanent molar is rare and usually occurs in the mandibular arch with an incidence of $0.06-0.3 \%[7,8]$. In our previous study, we found that the impaction of lower second molar -11 teeth $(4-2.2 \%$ on the right and $7-3.8 \%$ on the left) in 182 patient cases with impacted teeth. ${ }^{9}$ The lower second molar impaction is often detected in X-ray during the orthodontic treatment. Usually this problem is not the main reason for seeking orthodontic treatment. The early detection and the treatment is imperative to avoid a possible external root resorption of the first molar, caries and periodontal involvement of the first and second molars. Different authors offer methods of estimating probability of retention. ${ }^{10,11}$ The treatment of impaction second lower molars often requires the collaboration of orthodontist, oral surgeon and periodontist. The surgical treatment consists essentially of uprighting and repositioning of the second molar, eventually including extraction of the third molar. ${ }^{12,13}$

\section{Case presentation}

We present 22 years old female patient. The patient had basically aesthetic complaints from a crowding of the upper and lower frontal teeth. In the clinical examination, a shortened length of the lower dental arch was found on the left because a left lower second molar was impacted. Its antagonist tooth, upper second right molar, was over erupted, reaching the edge of the lower alveolar rouge. The over erupted upper left second molar blocked the occlusion. In the palate area of the upper first and second left molars was diagnosed a fibropapiloma. We recommended and conducted surgical removal of the fibroma. It was found, after the Orthopantomography that the impacted lower left second molar was strongly tipped medially and squeezed by the germ of third molar. We found a presence of four third molars. The orthodontic treatment plan included extraction of a lower left third molar, leveling the two dental arches, embracing the upper left second molar and straightening the lower left second molar. The patient refused extraction of the lower third molar and tooth extraction at all (Figure 1). The orthodontic guided traction and uprighting of the lower second molar had to be carried out in the same time with the influence on third molar. Mandibular second molars are usually impacted in the midalveolar region. Only one of the tubercles was visible, but the buccal, lingual and occlusal surfaces were completely covered with bone. The closest tooth (first left molar) was carious affected and not well restored. The unerupted second lower molar and the poorly restored first molar have created a condition for the growth of the antagonists, especially the upper second molar. This tooth was extruded more than 4-5 $\mathrm{mm}$ and has bowed down. This greatly hinders the downstream molar pull process. The orthodontic movements at the lower second molar are not possible until the upper left second molar is intruded, which will release space for the positioning of lower molar in the lower dental arch. The treatment started firstly with the intrusion of the upper left second molar, which made a space in vertical direction for the pooling of lower left second molar. The intrusion of 27 was carried out using micro implant palatinally for the support during the intrusion. The implant was lost and it was replaced with trans palatal arch - TPA and the individual loop (Figure 2). 


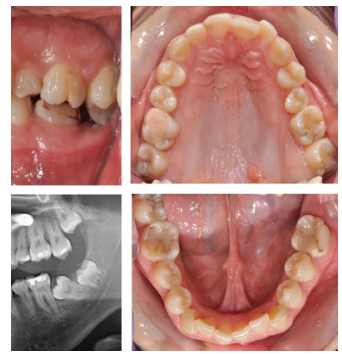

Figure I Initial situation of patient with impacted left lower second molar and over erupted antagonist - upper left second molar.

After leveling of the lower dental arch we used it as stabile support zone for a distal movement of second molar in the lower jaw (Figure 3). Initially, a bracket was bonded to the bucco-occlusal surface of the crown second molar, and a flexible wire was used to upright the tooth. Next then we used for distalization segment wire with loop. Then, a egment extrusion wire was placed gingival of the button bonded at second molar. We used a cantilever to upright the molar. Finally

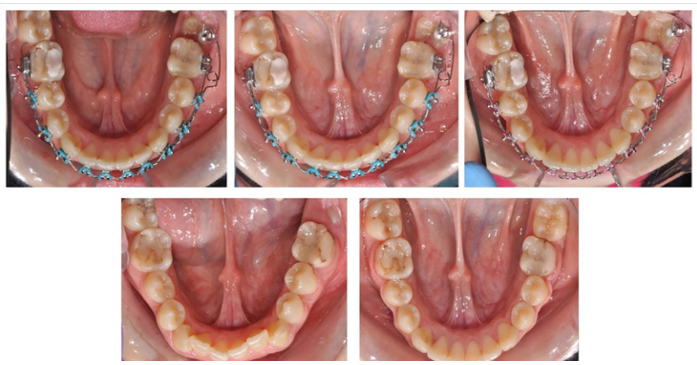

Figure 3 Extrusion and correct mesial inclination and vertical position of lower left second molar.

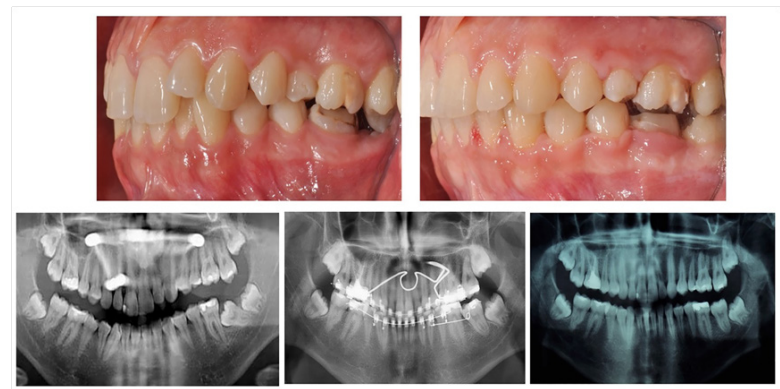

Figure 5 Progress the orthodontic treatment.

\section{Discussion}

The age for the lower second molar eruption is about 11-13 years. The later the problem is discovered, the more complicated is retrieval of the case. In our case, the additional complication was the over erupted upper antagonist. Without occlusion, the occlusion is blocked and freedom of movement of the lower jaw. The cheek effect of the dentition is impaired because the dental arch on the left is formed by only six teeth. An additional complication was the patient's desire not to extract the lower left third molar. The mini-implant for the support in the upper left first and second molar areas was lost. The patient said she habitually put her tongue on it because it was an area where the fibroma was previously, and she had a habit of putting
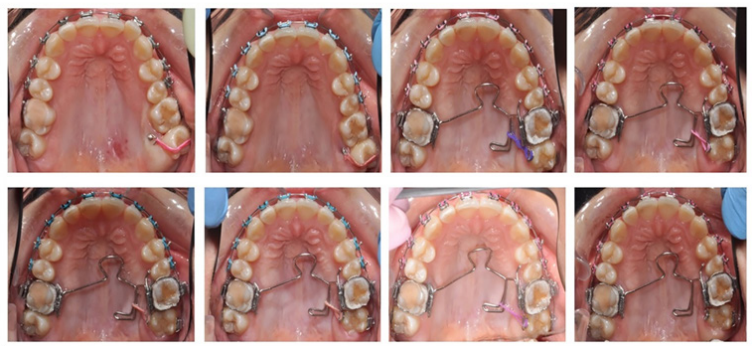

Figure $\mathbf{2}$ Intrusion process of the upper left second molar.

we bonded a canule and introduced second molar into the basic bracket wire for the complete leveling and finishing of the treatment. The effect of the treatment (Figure 4) was as follows: intrusion of upper second molar; uprighting and positioning the lower second molar in the dental arch; uprighting of lower third molar, but there a lack of space for the eruption of the third molar (Figure 5). Our recommending again to the patient was extraction of the third molars.
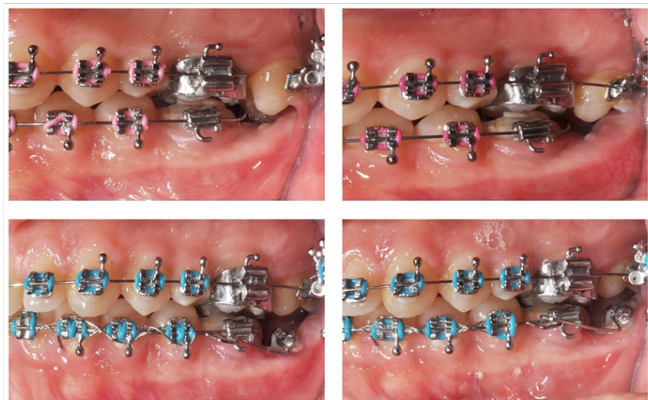

Figure 4 Change of occlusion during the treatment.

the tongue there. There was a necessity to change the conception of intrusion. Our decision was to use a modified trans-palatal arch to support the intrusion. The problem with the lower second molar was the need for movements in different directions: first, its straightening, then extrusion and finally leveling. That's why we changed several segmental arches with loops, which served the movements of lower second molar. The patient was pleased with the result achieved with which we restored aesthetics in the frontal segment as well as the function in the distal segment. It is crucial to make an early diagnosis, to choose optimal treatment time and reduction of complications. The mesial tipping of impaction and depth of impaction has more important roles than patient age and the difficulty of treatment.

\section{Acknowledgement}

None.

\section{Conflict of interest}

None.

\section{References}

1. Raghoebar G, Boering G, Vissink A, et al. Eruption disturbances of permanent molars: a review. J Oral Pathol Med. 1991;20(4):159-166.

2. Kjær I. Mechanism of Human Tooth Eruption: Review Article Including a New Theory for Future Studies on the Eruption Process. Hindawi Pub Corp Scien. 2014;13. 
3. Kenrad J, Vedtofte H, Andreasen J, et al. A retrospective overview of treatment choice and outcome in 126 cases with arrested eruption of mandibular second molars. Clin Oral Investig. 2011;15(1):81-87.

4. Raghoebar G, Boering G, Vissink A, et al. Eruption disturbances of permanent molars: a review. Journal of Oral Pathology \& Medicine. 1991;20(4):159-166.

5. Shpack N, Finkelstein T, Lai Y, et al. Mandibular Permanent Second Molar Impaction Treatment options and outcome. Open $J$ of Dent and Oral Med. 2013;1(1):9-14.

6. Shapira Y, Finkelstein T, Shpack N, et al. Mandibular second molar impaction Part I: Genetic trait and characteristics. Am J Orthod Dentofac Orthop. 2011;140(1):32-37.

7. Bondemark L, Tsiopa J (2007) Prevalence of ectopic eruption, impaction, retention and agenesis of the permanent second molar. Angle Orthod. 2007;77(5):773-778.

8. Po-Sung Fu, Wang JC, Wu YM, et al. Impacted mandibular second molars
A retrospective study of prevalence and treatment outcome. Angle Orthod. 2012;82(4):670-675.

9. Yordanova G, Stanimirov P. Impaction of teeth - frequency and treatment protocols. Stoma Edu J. 2017;4(4):53-60.

10. Baccetti T (2000) Tooth anomalies associated with failure of eruption of first and second permanent molar. Am J Orthod Dentofacial Orthop. 2000;118(6):608-610.

11. Alligri F, Putrino A, Cassetta M, et al. The mandibular permanent second molars and their risk of impaction: a retrospective study. Eur J of Paed Dent. 2015;16(3):246-250.

12. Lin S, Chang C, Roberts W. Simple Mechanics to Upright Horizontally Impacted Molars with Ramus Screws. Int J Orthod Impl. 2013;40:84-92.

13. Lau CK, Whang CZ, Bister D. Orthodontic uprighting of severely impacted mandibular second molars. Am J Orthod Dentofacial Orthop. 2013;143(1):116-124. 